\title{
Adoption of Electronic Personal Health Records in Canada: Perceptions of Stakeholders
}

\author{
Marie-Pierre Gagnon ${ }^{1,2,3^{*}}$, Julie Payne-Gagnon ${ }^{2}$, Erik Breton ${ }^{4}$, Jean-Paul Fortin ${ }^{3}$, Lara Khoury ${ }^{5}$, \\ Lisa Dolovich ${ }^{6}$, David Price ${ }^{6}$, David Wiljer ${ }^{7,8}$, Gillian Bartlett ${ }^{9}$, Norman Archer ${ }^{10}$
}

\begin{abstract}
Background: Healthcare stakeholders have a great interest in the adoption and use of electronic personal health records (ePHRs) because of the potential benefits associated with them. Little is known, however about the level of adoption of ePHRs in Canada and there is limited evidence concerning their benefits and implications for the healthcare system. This study aimed to describe the current situation of ePHRs in Canada and explore stakeholder perceptions regarding barriers and facilitators to their adoption.

Methods: Using a qualitative descriptive study design, we conducted semi-structured phone interviews between October 2013 and February 2014 with 35 individuals from seven Canadian provinces. The participants represented six stakeholder groups (patients, ePHR administrators, healthcare professionals, organizations interested in health technology development, government agencies, and researchers). A detailed summary of each interview was created and thematic analysis was conducted.

Results: We observed that there was no consensual definition of ePHR in Canada. Factors that could influence ePHR adoption were related to knowledge (confusion with other electronic medical records [EMRs] and lack of awareness), system design (usability and relevance), user capacities and attitudes (patient health literacy, education and interest, support for professionals), environmental factors (government commitment, targeted populations) and legal and ethical issues (information control and custody, confidentiality, privacy and security).

Conclusion: ePHRs are slowly entering the Canadian healthcare landscape but provinces do not seem wellprepared for the implementation of this type of record. Guidance is needed on critical issues regarding ePHRs, such as ePHR definition, data ownership, access to information and interoperability with other electronic health records (EHRs). Better guidance on these issues would provide a greater awareness of ePHRs and inform stakeholders including clinicians, decision-makers, patients and the public. In turn, it may facilitate their adoption in the country.

Keywords: Electronic Personal Health Record (ePHR), Adoption, Canada, Qualitative Research

Copyright: () 2016 by Kerman University of Medical Sciences

Citation: Gagnon MP, Payne-Gagnon J, Breton E, et al. Adoption of electronic personal health records in Canada: perceptions of stakeholders. Int J Health Policy Manag. 2016;5(7):425-433. doi: 10.15171/ijhpm.2016.36
\end{abstract}

Article History:

Received: 26 October 2015

Accepted: 2 April 2016

ePublished: 6 April 2016
*Correspondence to:
Marie-Pierre Gagnon
Email: marie-pierre.gagnon@fsi.ulaval.ca

\section{Key Messages}

Implications for policy makers

- It is important to use a clear and unique definition in communicating about electronic personal health records (ePHRs). Currently, it may be possible for two parties to talk about ePHRs while having totally different definitions of them. This article proposes a definition of ePHRs.

- Current Canadian legislation needs to be reviewed and adapted to the particular context of ePHRs, particularly with regard to custody of health information and privacy.

- Health and technology literacy issues were identified as important barriers to ePHR adoption. Patient education could lessen these issues and serve as a promotional tool for their engagement and awareness of ePHRs.

Implications for the public

Electronic personal health records (ePHRs) are seen as a solution that could facilitate patient access and control of their health-related information. However, ePHRs are still an uncommon sight in the Canadian healthcare information technology (IT) landscape and they are not well-known or widely used. This research explored stakeholders'-including patients representatives - perceptions of barriers and facilitators to ePHR adoption in the country, which may help the public by providing a better picture of the situation of ePHRs in Canada and a greater awareness of their existence and possibilities they offer. Greater awareness of ePHRs may further advance the cause of patient-centred care in the country and may promote patients' more active participation in their health. 


\section{Background}

In order to understand a patient's health issues, healthcare professionals gather objective and subjective information on that patient; yet little of that information is currently shared with patients. ${ }^{1}$ In the interest of patient access to their medical records, various legislation has been adopted worldwide, ${ }^{2}$ such as Europe's Data Protection Directive, ${ }^{3}$ USA's Health Insurance Portability and Accountability Act (HIPAA) ${ }^{4}$ or Canada's Personal Information Protection and Electronic Documents Act (PIPEDA). ${ }^{5}$ Even so, it remains problematic for patients to access their health records since access to copies of their medical record is often laborious. ${ }^{6}$ Consequently, it is harder for them to control their health-related information and to be involved in decisions and management related to their own health. ${ }^{1,6}$

On the other hand, patient access to health information is facilitated by the increased availability of information and communication technologies (ICTs). ${ }^{1,7}$ Among these, electronic personal health records (ePHRs) are seen as a solution that could be beneficial to facilitate access and control of a patient's health-related information, but also promote self-management of their health, improve patient safety, and enhance efficiency and effectiveness of patient care. $^{7-14}$ However, when using ePHRs, there are potential issues related to security and confidentiality, ${ }^{7,14-18}$ additional workload for professionals, ${ }^{2,8,19}$ health literacy issues among patients, technology literacy issues among users ${ }^{8}$ and the lack of awareness of ePHR existence. ${ }^{8,11}$ As there is no universally accepted definition of ePHRs, ${ }^{2}$ we propose a definition for this study based on two commonly used definitions of personal health records (PHRs). The first definition places the emphasis on the patient view of information contained in medical or health records. In this definition, PHRs are seen as a set of tools that offer the patient a comprehensive view of his or her health information that may include information entered by them, but also test results, diagnoses, medication or other information from healthcare professionals. The second definition ${ }^{20}$ puts control and management of the information at the centre of the definition. Indeed, ePHRs are defined as a an array of tools allowing patients access to health information added by them, their advocates and their healthcare professionals, and empowering their participation in their own care by providing them with complete control over that information.

Thus, our definition of ePHRs is a set of electronic tools providing patients with a comprehensive view of and timely access to their health information, entered and maintained by them and/or their healthcare professionals, and allowing patients to be custodians of their information and exert a certain amount of control over it (from deciding who can access their information to fully controlling the information), as well as giving them the ability to manage, track, and participate in their own healthcare.

We distinguish four categories of ePHRs that can be encompassed in this definition. First, the stand-alone ePHR allows the collection of health information on a portable media device or a website that generally enables viewing and managing the data by the patient only. ${ }^{11}$ Second, the tethered ePHR is managed by a facility or institution. Patients can access and update their health information from the facility or institution's electronic medical record (EMR) or electronic health record (EHR) with various degrees of control. ${ }^{21}$ Third, an untethered ePHR does not communicate with an EMR or an EHR, but allows patients and sometimes the healthcare providers to have access to the record. Finally, the integrated ePHR makes it possible to gather and view data from multiple sources, such as an EHR and/or an EMR, patients, or healthcare providers. ${ }^{11}$

In Canada, e-health has been progressing slowly. ${ }^{10,15,22}$ Among other factors, this slow progress has been caused by technological issues, lack of governmental support and budget overruns. ${ }^{10,23}$ Consequently, the implementation of accessible electronic records - such as ePHRs - is also progressing slowly. ${ }^{10,24}$ Indeed, there are few ePHRs available for the Canadian population and they are neither widely known, nor used. ${ }^{25-35}$ Moreover, there is no ePHR-specific legislation or regulation in Canada. ${ }^{36}$ There are, however, legislation and regulations that deal with EHRs that might provide relevant inspiration when designing rules governing the implementation of ePHRs, even if their direct applicability to ePHRs is often doubtful. ${ }^{36,37}$

Nevertheless, healthcare stakeholders have a great interest in the adoption and use of ePHRs, because of the potential benefits associated with them. ${ }^{8-13,38}$ However, little is known about the current use and attitudes towards ePHRs in Canada and there is limited scientific evidence concerning their benefits and implications for healthcare systems. ${ }^{8,9,39}$ In order to fill this gap, we conducted a study that explored stakeholders' perceptions of ePHRs and their views of barriers and facilitators to adoption in Canada.

\section{Methods \\ Study Design and Population}

We used a qualitative descriptive design in order to provide rich descriptions of ePHRs in Canada and to improve understanding of the topic. We employed a purposive sampling approach, targeting individuals who were known as Canadian experts on health records and health information accessibility or members of patient groups. These persons were first identified as experts by the members of the research team. The list was developed from team members' contacts with experts in the domain and through the scientific literature. Using the snowball technique, the experts that were recruited also pointed out other experts in the domain that were subsequently contacted and interviewed. Three members of the research team (EB, JPF, and JPG) contacted 39 experts by email or by phone based on the contact information available. The CHU de Québec Research Centre ethics board reviewed the study protocol and deemed it not necessary to seek ethical approval for this study since participants were considered as experts. However, the study adheres to the usual ethical considerations related to privacy, confidentiality and data management in research.

\section{Data Collection}

Two members of the team (EB and JPG) conducted semistructured interviews by phone between October 7, 2013, and February 11, 2014. The interviewees determined the interview schedule according to their availability. Therefore, most of the interviews took place at their workplace or their 
home. Interviews were conducted in either French or English. At the beginning of each interview, the interviewer took the time to differentiate an EMR from an ePHR using a shortened version of the aforementioned definition in order to ensure that the interviewer and the interviewee would be discussing the same topic. Contrary to ePHRs, EMRs belong to a healthcare provider's practice or organization and healthrelated information is entered in this type of record by the healthcare provider only. ${ }^{40}$ The interview guide consisted of three main themes: participants' point of view about ePHRs, perceived benefits or hindrances of using ePHRs, and barriers and facilitators to the adoption of this technology. The interview guide is available in Appendix 1. The interviews were audio recorded with the participants' consent (received verbally before the recording).

\section{Analysis}

A detailed summary of each interview was created. We analysed data following the method described by Miles and Huberman, which consists of three steps: data reduction (identification of the main themes), data display (organized classification of data) and drawing conclusions (results explanation). EB and JPG independently performed the analysis with the support of NVivo 8, a qualitative data analysis software package. ${ }^{41}$ Codification was an iterative process and was based on themes emerging from the interviews.

\section{Results}

Characteristics of the Participants

Of the 39 potential participants, four did not reply to our invitation, bringing the total number of participants to 35. Twenty-nine interviews were with individual people and three interviews were conducted jointly with two participants. Interview duration averaged eighteen minutes, and 59 pages of summarized transcript were produced. The participants represented six stakeholder groups: patients, ePHR administrators, healthcare professionals (nurses and physicians), organizations interested in health technology assessment and development, government health agencies, and researchers. The most represented group was patients with 14 representatives (40\%), while three to five participants represented other groups. Genders were represented equally (18 females and 17 males). The majority of the participants (82.9\%) came from the provinces of Quebec (15 participants) and Ontario (14 participants). Five other provinces (British Columbia, Alberta, New Brunswick, Nova Scotia, and Saskatchewan) accounted for six participants. See Table 1 for more details on participant characteristics.

For presentation purposes, the results section will report on 32 interviews, as we counted interviews with two participants as one unique entry since the participants were in agreement with each other and made similar or complementary comments during the interviews. See Table 2 for a summary of the most important adoption themes identified by the participants in regards to ePHRs and Appendix 2 for selected quotes from the interviews for each theme.

\section{Knowledge on Electronic Personal Health Records}

We noticed that 22 participants had a view of ePHRs corresponding to only one of the two dimensions of the
Table 1. Characteristics of the Participants Interviewed

\begin{tabular}{|c|c|c|}
\hline \multirow[t]{2}{*}{ Participants' Characteristics } & \multicolumn{2}{|c|}{$\begin{array}{c}\text { All Participants } \\
\mathrm{n}=35\left(32^{\mathrm{a}}\right)\end{array}$} \\
\hline & Number & $\%$ \\
\hline \multicolumn{3}{|l|}{ Group (representatives) } \\
\hline Patients & 14 & 40.000 \\
\hline ePHR administrators & $5(3)$ & 14.286 \\
\hline Health professionals & $5(4)$ & 14.286 \\
\hline Health technology organizations & 5 & 14.286 \\
\hline Government health agencies & 3 & 8.571 \\
\hline Researchers & 3 & 8.571 \\
\hline \multicolumn{3}{|l|}{ Gender } \\
\hline Female & 18 & 51.400 \\
\hline Male & 17 & 48.600 \\
\hline \multicolumn{3}{|l|}{ Province } \\
\hline Quebec & $15(13)$ & 42.850 \\
\hline Ontario & $14(13)$ & 40.000 \\
\hline British Columbia & 2 & 5.710 \\
\hline Alberta & 1 & 2.860 \\
\hline New Brunswick & 1 & 2.860 \\
\hline Nova Scotia & 1 & 2.860 \\
\hline Saskatchewan & 1 & 2.860 \\
\hline
\end{tabular}

all numbers in parentheses indicate the total number of participants in two-person interviews counted as only one participant.

definition that we presented in the introduction: information visualization by the patient (11) or patient management of the information (11). Participant group representatives were torn between these two dimensions, with the exception of government officials who were exclusively interested in the visualization dimension of ePHRs. Also, eight participants from the patient, government and professional groups confused ePHRs with electronic medical records (EMRs). At first, these participants believed that the interview was about EMRs even if the term ePHR was explicitly used in the recruitment email. Moreover, except for those directly involved in an ePHR project, participants seemed to perceive ePHRs as a type of record or idea to potentially implement, rather than as a type of record currently available. Indeed, most spoke of the potential benefits associated with the use of ePHRs and of the possible facilitators, barriers or repercussions to their implementation and use in Canada.

\section{System Design}

Seventeen participants from all groups mentioned simplicity of the language used, availability of the information, system reliability and ease of access as design options that could improve usability and relevance of ePHRs. They also identified attractive layout, customization available to cater to patient needs, mobility and technology that withstands technological advancements as other interesting options to improve usability. However, the most recurrent facilitator identified was ease of use, mentioned by six participants. In particular, they mentioned that ePHRs should be easy to understand and navigate for all user groups, as well as providing support to correct the glitches they may encounter.

Four patient representatives and one professional perceived interoperability as an important issue that would ensure efficient sharing of information between different healthcare professionals and the patient. However, they explained that interoperability between different records is currently almost 
Table 2. Main Adoption Themes Identified by the Participants

\begin{tabular}{|c|c|c|c|c|c|c|c|}
\hline Themes & $\begin{array}{c}\mathbf{n} \\
\text { Total }\end{array}$ & $\begin{array}{c}\mathbf{n} \\
\text { Patient }\end{array}$ & $\stackrel{n}{\text { Administrator }}$ & $\begin{array}{c}n \\
\text { Professional }\end{array}$ & $\begin{array}{c}\mathbf{n} \\
\text { Technology } \\
\text { Organization }\end{array}$ & $\begin{array}{c}n \\
\text { Government }\end{array}$ & $\begin{array}{c}n \\
\text { Research }\end{array}$ \\
\hline \multicolumn{8}{|l|}{ Knowledge about ePHRs } \\
\hline Confusion between EMRs and ePHRs & 8 & 6 & - & 1 & - & 1 & - \\
\hline \multicolumn{8}{|l|}{ System design } \\
\hline Design options to improve use of ePHRs (multiple) & 17 & 5 & 1 & 1 & 5 & 2 & 3 \\
\hline Lack of interoperability & 5 & 4 & - & 1 & - & - & - \\
\hline Digital divide & 5 & 4 & - & - & 1 & - & - \\
\hline \multicolumn{8}{|l|}{ User capacities and attitudes } \\
\hline Lack of health literacy & 14 & 7 & 1 & 1 & 1 & 2 & 2 \\
\hline Professional reluctance to use ePHRs (multiple) & 12 & 5 & 1 & 1 & 3 & 2 & - \\
\hline Support of ePHRs by professionals & 9 & 2 & 3 & 1 & 2 & - & 1 \\
\hline Lack of IT literacy & 8 & 4 & - & 1 & 1 & 1 & 1 \\
\hline Patient education & 8 & 4 & - & 1 & 3 & - & - \\
\hline \multicolumn{8}{|l|}{ Environmental factors } \\
\hline Government commitment to ePHRs & 8 & 2 & 2 & - & 2 & 1 & 1 \\
\hline Emphasis on patients with chronic diseases & 8 & 2 & - & 1 & 4 & - & 1 \\
\hline Lack of governmental policies on ePHRs & 6 & 1 & 1 & 2 & 1 & 1 & - \\
\hline Variable level of e-health implementations & 5 & 2 & - & - & 1 & 1 & 1 \\
\hline \multicolumn{8}{|l|}{ Legal and ethical issues } \\
\hline Confidentiality, privacy and security issues & 29 & 14 & 1 & 4 & 5 & 2 & 3 \\
\hline Control and custody of information (barrier and facilitator) & 18 & 8 & 2 & 3 & 3 & 1 & 1 \\
\hline Outdated provincial legislation & 5 & - & - & 2 & 2 & 1 & - \\
\hline
\end{tabular}

Abbreviations: ePHR, electronic personal health record; EMR, electronic medical record.

non-existent, which is impeding information access. A government official and a professional added that using preexisting systems (eg, EMRs or provincial health information systems) could facilitate ePHR implementation and reduce costs since there would be a basis to build on. Finally, four patients and one information technology (IT) specialist raised issues concerning the "digital divide," namely limited access to a computer or the Internet. The most disadvantaged people may not have access to these technologies and consequently, may have restricted or no use of ePHRs.

\section{User Capacities and Attitude}

Comments related to user capacities and attitudes concerned the two main groups of ePHR users: patients and healthcare professionals. First, patient health literacy was seen as a major issue for ePHR usability by 14 participants. As they explained it, the lack of comprehension of healthcare information contained in a record could cause misinterpretation of the data, provoke incorrect entry of data, and overwhelm the user with too much information to handle. Eight participants from all groups but ePHR administrators also noted that the lack of IT literacy among patients could cause problems understanding how to use computers or accessing the system. More particularly, two patients and one IT specialist were worried about an important cross-section of potential users of ePHRs, the elderly, who generally are less knowledgeable about technologies. As suggested by eight participants, educating patients could lessen health literacy issues and serve as a promotional tool for patient engagement and awareness of ePHRs and their uses. IT literacy issues could also be lessened by patient education and by implementing some system design options presented earlier. Another potential barrier mentioned by three patient representatives was patient interest in ePHRs: their interest could be low if they did not perceive added value from using ePHRs - such as access to their information or enhanced control over it - or if they had no desire to be accountable for their own health and would prefer that their healthcare professionals be in charge of it. Nine participants from all groups except the government deemed that support, openness and interest by healthcare professionals in taking time to read information contained in ePHRs was critical to their successful use. Indeed, these factors were often perceived as an important element for adoption of ePHRs since physicians' support would ensure greater sustainability of ePHR use and a more favourable opinion of ePHRs by patients. Conversely, 12 participants feared that professionals would be reluctant to use ePHRs. The reasons that were mentioned were the potential increased workload related to the use of the system, the fear of sharing information with the patient because they might be anxious over unexplained data or could not understand it due to their lack of literacy. In addition, the lack of credibility of patiententered data could cause problems since patients could potentially hide, modify or add incomplete or inaccurate information in their record.

\section{Environmental Factors}

Five participants from all groups but professionals and ePHR administrators noted that since health falls under provincial jurisdiction in Canada, each province has a different level of e-health implementation, possibly leading to a variable interest in ePHRs. A researcher also specified that in many Canadian jurisdictions, complete health information is not available electronically to healthcare professionals. Consequently, it is even less available to patients. Until all information is available electronically, this will remain a major hurdle to putting electronic records in place. Moreover, six participants from all groups felt that government policies 
are relatively silent on many aspects of patient-centred care. Considering that eight participants believed that provincial governments need to establish goals and commit themselves to facilitate ePHR adoption, this may pose a challenge to their adoption. Unsurprisingly, ePHR administrators agreed with government investment in ePHRs, but disagreed with other groups of participants on their management, preferring the management of ePHRs be done by their respective organizations instead of government agencies.

Eight participants believed that some patient populations may be more interested in ePHRs than others. These included patients with chronic diseases (all eight participants), the elderly people (IT specialist), young mothers (IT specialist) and persons with reduced mobility (professional). Two IT specialists and a patient specified that the main opportunity for governments would be to target patients with chronic diseases, since they are the greatest users of the healthcare system and ePHRs could greatly facilitate the management of their condition. Two IT specialists and a patient, however, believed that there were currently too few government incentives to promote self-management among this population, because of lack of financial support among other issues.

Another potential barrier to ePHR adoption was the pricing model chosen. Two IT specialists, one patient and one researcher feared patients would be reticent to using an ePHR if it involved paying usage fees. Indeed, some patients may believe that they already pay sufficiently for healthcare and should not be charged even more for the use of an ePHR. Moreover, some disadvantaged people might not even have the financial resources to pay for the usage of such a record. On the other side of the spectrum, two ePHR administrators and one IT specialist mentioned that support to healthcare professionals through remuneration for promoting and using ePHRs could be a good way to encourage their general adoption. Interestingly, the other groups (patients and professionals, respectively) did not comment on these issues.

\section{Legal and Ethical Issues}

Eighteen participants had widely polarized views on the subject of control and management of ePHR information. Among these, one representative from each group believed that access should be granted to the healthcare professionals who need it, according to predetermined rules. On the other hand, six patient representatives, one IT specialist and one ePHR administrator believed that patients would have reticence in giving professionals or other parties access to all the information contained in their records and would prefer that the information be shared with their consent only. Five participants from the professional, patient, researcher, and government groups were cautious when discussing patient control of the data. As explained earlier, they feared the information could be less credible or complete, particularly in cases of complete patient control over their record. Also, three patient representatives were worried that government, insurance companies or family members could interfere with the record, consequently violating the owner's privacy. Other groups did not mention this issue.

The most often mentioned issues related to ePHR implementation pertained to confidentiality, privacy and security of information. The majority (29 participants) mentioned at least one of these three terms during the interview. While we acknowledge that these terms have different meanings, they were so often bundled together that we decided to regroup them in a single section. This was particularly the case for confidentiality and privacy. The participants used these two terms interchangeably, suggesting that they considered them equivalents. Security was less of a problem in this respect, since it was perceived as a means to attain confidentiality or privacy. In general, participants believed that confidentiality and privacy of information should be ensured through secured access to ePHRs. Also, two participants from Ontario and three from Quebec believed that their respective provincial legislation related to these matters was outdated and needed to be better adapted to ePHRs.

\section{Discussion}

The objective of this study was to explore stakeholder perceptions regarding ePHR adoption in Canada and the benefits from their use. Overall, the factors identified in this study were mentioned at least by two groups of participants. Among these factors, some recurred more often than others. Usability of the record, support from healthcare professionals, patient health and IT literacy, provincial government commitment, security, privacy, confidentiality, as well as control and custody of the information, were aspects that many participants raised as important factors that affect ePHR adoption.

There were few differences identified between the groups. Only patient representatives mentioned the importance of patient interest in ePHR. They were also the main group of experts believing that ePHR information should be shared with the patient's consent. These findings are not surprising considering that patient representatives are often defending patients' interests and are particularly sensitive about factors affecting them. ${ }^{42}$ ePHR administrators thought that the management of ePHR should be done by organizations other than the government, a point of view that differed from that of other groups. Again, this is not surprising since these representatives generally have their organization's interest in mind.

Many factors raised by the participants are also prevalent in the scientific literature. Usability, ${ }^{16,43,44}$ support from professionals, ${ }^{7}$ targeting specific population of users, ${ }^{1,8,12,25,45}$ lack of trust in patient-generated data, ${ }^{38,46,47}$ digital divide, ${ }^{16,48}$ literacy issues, ${ }^{25,47}$ increased workload for professionals, ${ }^{8,25}$ and privacy, confidentiality and security $y^{7,9,15,16,18}$ are among these recurrent factors. However, this study also identifies issues that are less common in the literature or brought interesting subtleties to some known issues: emphasis on patients with chronic diseases, custody and control of these records, legislation related to ePHRs, and the different points on the definition of ePHRs.

As seen through the interviews and in the literature, patients with chronic diseases are perceived as an important group to be considered in ePHR adoption. ${ }^{1,8,25,45,49}$ As the greatest users of the healthcare system, they have a more significant interest in self-management of their health and in accessing their health information than any other group of users. ${ }^{1,49}$ Moreover, if access to their own health information for 
patients with chronic disease continues to be restricted, it is estimated that costs for the healthcare system will continue to escalate.

Some legal and ethical issues also need to be considered for ePHR adoption. First, there are divergent opinions on control and management of the information, which may be partly explained by the legal treatment of custody of health information in Canada. While the content of a record is owned by the patient, the physical record itself is owned by the professional. ${ }^{47}$ This classification may divide opinions on the subject of who has custody of information. Second, there is overlapping constitutional jurisdiction over privacy in Canada. Legislation exists at both the federal and provincial levels and many provinces possess separate statutes and regulations dealing with privacy for the private, public, and healthcare sectors. ${ }^{50}$ This multiplicity of relevant statutes and regulations may complicate one's understanding of the legal framework governing ePHRs in Canada. Third, there is currently no regulation or legislation specific to ePHRs in the country, which may complicate even more the current legal and ethical framework surrounding this type of record.

Also, the lack of consensus on the definition of ePHRs may seriously impact their implementation. Since it may be possible for two parties to talk about ePHRs while having totally different definitions of them, partnerships, management and policy-making may be more difficult. ${ }^{51}$ It is thus important to use a clear and unique definition in communicating about ePHRs. Additionally, most of the participants' comments were comprised of words such as "may," "could," "should," or "idea" when talking about ePHRs. This may result from the fact that ePHRs are often not a reality in the healthcare field since they are almost absent from the landscape in most Canadian provinces. There are only some ePHRs available in the country and they are not well-known or widely used. ${ }^{25-35}$ This situation makes it difficult for participants to discuss concrete factors influencing ePHR adoption and use.

\section{Limitations}

There were some limitations to this study. First, confusion between the definitions of EMRs and ePHRs was an impediment to the study, even if we verified that every participant knew the difference between the two types of records at the beginning of each interview. We ascertained, however, that all data applied to ePHRs in our analysis. Participants from the patient group represented almost half of the total participants in this study. A better balance among the groups might have resulted in more diverse data being collected. Nonetheless, we believe that data saturation was achieved among the patients. Even if it could be interesting to complement the results with additional comments from the other groups, their points of view were consistent with those from patients and in line with general comments on the topic. Finally, two provinces were over-represented in this study: Ontario and Quebec, accounting for more than $80 \%$ of the participants. As our research project focuses specifically on these two provinces, a greater effort was put on the recruitment of representatives from these provinces, explaining this disproportional recruitment. As ePHR projects are also being implemented in other provinces, such as Alberta, Saskatchewan, and Nova Scotia, it would be important to involve more representatives from these provinces in the future in order to get a more nuanced view of the current situation of ePHRs in Canada.

\section{Conclusion}

ePHRs have the potential to facilitate access to healthrelated information, improve care, promote more active patient participation in their health and further the cause of patient-centred care in the country. However, they are still an uncommon sight in the Canadian healthcare IT landscape. Too few professionals and patients are aware of their availability and this was noticeable even among experts interviewed. Additionally, Canada does not seem well-prepared for the implementation of ePHRs since many provinces are lagging behind on e-health technologies and current Canadian legislation needs to be reviewed and adapted to the particular context of ePHRs. Guidance is needed on ePHR definition, data ownership, access to information and interoperability with other health records since these represent critical issues that impede the progression of ePHRs in Canada. In conjunction with a greater awareness of ePHRs, better guidance and policy adoption on these issues may facilitate their adoption in the country.

\section{Acknowledgements}

This work was supported by a grant from the Canadian Institutes of Health Research, Ottawa, ON, Canada (201209HIC 298617-HRA-CFBA-111141). The Canadian Institutes of Health Research had no role in the design and conduct of the study, data collection, data management, data analysis and interpretation, preparation, review or approval of the manuscript.

\section{Ethical issues}

The CHU de Québec Research Centre, Quebec City, QC, Canada ethics board deemed not necessary to seek ethical approval for this study.

\section{Competing interests}

Authors declare that they have no competing interests.

\section{Authors' contributions}

MPG, JPF, LK, LD, DP, DW, GB, and NA contributed to the study concept and design. MPG supervised the study. EB, JPG, and JPF recruited the participants. EB and JPG conducted the interviews as well as the analysis and interpretation of data. MPG and JPG drafted the manuscript. All of the authors critically revised the manuscript for important intellectual content and approved the final version submitted for publication.

\section{Authors' affiliations}

${ }^{1}$ Faculty of Nursing Science, Université Laval, Quebec City, QC, Canada. ${ }^{2}$ Research Centre of the CHU de Québec-Université Laval, Quebec City, QC, Canada. ${ }^{3}$ Centre de recherche sur les soins et les services de première ligne, Centre intégré de santé et services sociaux de la Capitale-Nationale, Quebec City, QC, Canada. ${ }^{4}$ Faculty of Medicine, Université Laval, Quebec City, QC, Canada. ${ }^{5}$ Faculty of Law, McGill University, Montreal, QC, Canada. ${ }^{6}$ Department of Family Medicine, McMaster University, Hamilton, ON, Canada. ${ }^{7}$ Institute of Health Policy, Management and Evaluation, University of Toronto, Toronto, ON, Canada. ${ }^{8}$ Centre for Addiction and Mental Health, Toronto, ON, Canada. 9Department of Family Medicine, McGill University, Montreal, QC, Canada. ${ }^{10}$ DeGroote School of Business, McMaster University, Hamilton, ON, Canada.

\section{References}

1. Leonard KJ, Casselman M, Wiljer D. Who will demand access to their personal health record? a focus on the users of health 
services and what they want. Healthc Q. 2008;11(1):92-96. doi:10.12927/hcq.2008.19503

2. Wiljer D, Urowitz S, Apatu E, et al. Patient accessible electronic health records: exploring recommendations for successful implementation strategies. J Med Internet Res. 2008;10(4):e34. doi:10.2196/jmir.1061

3. European Commission. Protection of personal data. http:// ec.europa.eu/justice/data-protection/.http://ec.europa.eu/justice/ data-protection/. Updated 2016. Accessed January 22, 2016.

4. Solove DJ. HIPAA Turns 10: Analyzing the Past, Present, and Future Impact. J AHIMA. 2013;22:23-28.

5. Peekhaus W. Personal health information in Canada: A comparison of citizen expectations and legislation. Gov Inform Q. 2008;25(4):669-698. doi:10.1016/j.giq.2007.05.002

6. Carrión Señor I, Fernández-Alemán JL, Toval A. Are personal health records safe? A review of free web-accessible personal health record privacy policies. J Med Internet Res. 2012;14:e114. doi:10.2196/jmir.1904

7. Archer NP, Cocosila M. Canadian Patient Perceptions of Electronic Personal Health Records: An Empirical Investigation. Communications of the Association for Information Systems. 2014;34:20.

8. Archer N, Fevrier-Thomas U, Lokker C, McKibbon KA, Strauss SE. Personal health records: a scoping review. J Am Med Inform Assoc. 2011;18(4):515-522. doi:10.1136/amiajnl-2011-000105

9. Williams JB, Weber-Jahnke JH. The regulation of personal health records in Canada. Can J Law Technol. 2010;8(2):241274.

10. Urowitz S, Wiljer D, Apatu E, et al. Is Canada ready for patient accessible electronic health records? A national scan. BMC Med Inform Decis Mak. 2008;8:33. doi:10.1186/1472-6947-8-33

11. Assadi V. Adoption of Integrated Personal Health Record Systems: A Self-Determination Theory Perspective [PhD thesis]. Hamilton: McMaster University; 2013.

12. Daglish D. Electronic Personal Health Records: A Matter of Trust [PhD thesis]. Hamilton: McMaster University; 2013.

13. Alberta Health Services. Engaging the Patient in Healthcare: An Overview of Personal Health Record Systems and Implications for Alberta. Edmonton: Alberta Health Services; 2009.

14. de Lusignan S, Mold F, Sheikh A, et al. Patients' online access to their electronic health records and linked online services: a systematic interpretative review. BMJ Open. 2014;4(9):e006021. doi:10.1136/bmjopen-2014-006021

15. Pai HH, Lau F, Barnett J, Jones S. Meeting the health information needs of prostate cancer patients using personal health records. Curr Oncol. 2013;20(6):e561-e569. doi:10.3747/co.20.1584

16. Emani S, Yamin CK, Peters E, et al. Patient perceptions of a personal health record: A test of the diffusion of innovation model. J Med Internet Res. 2012;14(6):e150.

17. Lafky DB, Horan TA. Personal health records: Consumer attitudes toward privacy and security of their personal health information. Health Inform J. 2011;17(1):63-71. doi:10.1177/1460458211399403

18. Luque AE, van Keken A, Winters P, Keefer MC, Sanders M, Fiscella K. Barriers and facilitators of online patient portals to personal health records among persons living with HIV: formative research. J Med Internet Res. 2013;15(1):e8. doi:10.2196/ resprot. 2302

19. Cahill JE, Gilbert MR, Armstrong TS. Personal health records as portal to the electronic medical record. J Neurooncol. 2014;117(1):1-6. doi:10.1007/s11060-013-1333-x

20. The Personal Health Working Group. Connecting for Health: A Public-Private Collaborative. New York: Markle Foundation; 2003.

21. Skinner HH, Price DJ, Tatham W. Connected Health \& Wellness
Project Final Report. Canada: Connected Health \& Wellness Project; 2014

22. Mynors G, Newsom-Davis E. Guide to Health Records Access. United Kingdom: Patient Information Forum; 2012.

23. Rozenblum R, Jang Y, Zimlichman E, et al. A qualitative study of Canada's experience with the implementation of electronic health information technology. Can Med Assoc J. 2011;183(5):e281288. doi:10.1503/cmaj. 100856

24. Webster PC. Albertans to gain electronic access to personal health files. Can Med Assoc J. 2010;182(10):e431-e432. doi:10.1503/cmaj.109-3270

25. Miriovsky BJ, Shulman LN, Abernethy AP. Importance of health information technology, electronic health records, and continuously aggregating data to comparative effectiveness research and learning health care. J Clin Oncol. 2012;30(34):4243-4248. doi:10.1200/jco.2012.42.8011

26. Stylus Consulting. Nova Scotia Personal Health Record Demonstration Project: Benefits Evaluation Report. Toronto: Stylus Consulting; 2014.

27. Alberta Health Services. eHealth and your patients. https:// myhealth.alberta.ca/eHealthforProviders/Pages/default.aspx. Updated 2014. Accessed February 11, 2016.

28. McMaster University Department of Family Medicine. MyOscar: Your Connection To Health. http://myoscar.org/. Updated 2011. Accessed February 11, 2016.

29. McGill Comprehensive Health Improvement Program. MyHealthCheckup website. http://myhealthcheckup.com/. Updated 2014. Accessed February 11, 2016.

30. Sunnybrook Health Sciences Centre. MyChart. http:// sunnybrook.ca/content/?page=mychartlogin-learnmore. Updated 2016. Accessed February 11, 2016.

31. Canadian Medical Association. Mydoctor.ca Health Portal. https://www.mydoctor.ca/patient/welcome.do. Updated 2016. Accessed February 11, 2016.

32. Nightingale. My Patient Access: My patient. My Physician. My Access. http://www.mypatientaccess.ca/. Updated 2015. Accessed February 11, 2016.

33. McKesson Canada. RelayHealth website. http://www. relayhealth.ca/. Updated 2015. Accessed February 11, 2016.

34. Telus Health. Telus health space website. http://telushealthspace. com/. Updated 2013. Accessed February 11, 2016.

35. Mihealth Global Systems. Mihealth website. https://www. mihealth.com/. Updated 2016. Accessed February 11, 2016.

36. My Digital Primary Health Care. My Digital Primary Health Care. http://www.mapremierelignenumerique.ca/en/. Updated 2015. Accessed February 11, 2016.

37. Khoury L, Horwood F, Gagnon MP. Legal Norms and Public Policies Impacting ePHR Implementation. Montreal: McGill University; 2014.

38. Cushman R, Froomkin AM, Cava A, Abril P, Goodman KW. Ethical, legal and social issues for personal health records and applications. J Biomed Inform. 2010;43(5):S51-S55. doi:10.1016/j.jbi.2010.05.003

39. Piras EM, Zanutto A. "One day it will be you who tells us doctors what to do!" Exploring the "Personal" of PHR in paediatric diabetes management. Inform Tech People. 2014;27(4):421439.

40. Goldzweig CL, Orshansky G, Paige NM, et al. Electronic patient portals: evidence on health outcomes, satisfaction, efficiency, and attitudes: a systematic review. Ann Intern Med. 2013;159(10):677-687.

41. Canada Health Infoway. Electronic Medical Record (EMR). https://www.infoway-inforoute.ca/en/home/82-our-partners/ vendors/certification-services/240-emr. Updated 2016. Accessed February 11, 2016. 
42. Miles MB, Huberman AM. Qualitative Data Analysis: An expanded Sourcebook. 2nd ed. Thousand Oaks: SAGE Publications; 2003.

43. QSR International. Qualitative Research, Data Analysis Software, NVivo. http://www.qsrinternational.com/. Updated 2016. Accessed February 11, 2016.

44. Gagnon MP, Desmartis M, Gagnon J, et al. Framework for user involvement in health technology assessment at the local level: Views of health managers, user representatives, and clinicians. Int J Technol Assess Health Care. 2015;31(1-2):6877. doi:10.1017/S0266462315000070

45. Goel MS, Brown TL, Williams A, Cooper AJ, Hasnain-Wynia $\mathrm{R}$, Baker DW. Patient reported barriers to enrolling in a patient portal. J Am Med Inform Assoc. 2011;18:i8-i12. doi:10.1136/ amiajnl-2011-000473

46. Britto MT, Hesse EA, Kamdar OJ, Munafo JK. Parents' perceptions of a patient portal for managing their child's chronic illness. J Pediatr. 2013;163(1):280-281. doi:10.1016/j. jpeds.2013.02.041

47. Hordern A, Georgiou A, Whetton S, Prgomet M. Consumer e-health: an overview of research evidence and implications for future policy. HIM J. 2011;40(2):6-14. doi:10.1177/183335831104000202

48. Storni C. Diabetes self-care in-the-wild: Design challenges for personal health record systems and self-monitoring technologies. Inform Tech People. 2014;27(4):397-420. doi:10.1108/ITP-022013-0032

49. Day K, Gu Y. Influencing factors for adopting personal health record (PHR). Stud Health Technol Inform. 2012;178:39-44.

50. Yamin CK, Emani S, Williams DH, et al. The digital divide in adoption and use of a personal health record. Arch Intern Med. 2011;171(6):568-574.

51. Tenforde M, Jain A, Hickner J. The value of personal health records for chronic disease management: What do we know? Fam Med. 2011;43(5):351-354.

\section{Appendix 1. Interview Guide \\ Point of view on ePHRs}

1. What is the point of view of your organisation about electronic access of patient's personal health records (PHRs)?

\section{Benefits and inconveniences to ePHRs use}

2. What are the principal benefits associated with the use of electronic personal health record (ePHR) (for patients, for physicians and other healthcare professionals)?

3. Are they some risks or inconveniences linked to the use of ePHR (for patients and relatives, physicians, other healthcare professionals)? What are they?

\section{Barriers and facilitators to ePHR adoption}

4. What are the factors that could facilitate the use of ePHR (for the patients, their relatives, the healthcare professionals)?

5. Are there elements that may limit the use of ePHR (for patients, physicians)? What are they?

6. In your opinion, what are the legal factors that can influence the implementation or use of ePHR?

7. In your opinion, how governmental policies can influence the implementation or use of ePHR? 
Appendix 2. Adoption Themes Identified and Selected Quotes From the Participants

\begin{tabular}{ll}
\hline Related Theme & Quote \\
\hline $\begin{array}{l}\text { Knowledge about ePHRs } \\
\text { Confusion between EMRs and ePHRs }\end{array}$ & $\begin{array}{l}\text { Patient: In hospitals, there are files shelves next to waiting rooms. Electronic records may be a danger, an } \\
\text { inconvenience, but I do not think that it is greater than paper files. } \\
\text { Interviewer: It seems to me that you talk about EMRs. I want to reaffirm that we talk about another system, } \\
\text { ePHRs. }{ }^{\text {a }}\end{array}$ \\
$\begin{array}{ll}\text { System design } \\
\text { Design options to improve use of ePHRs } \\
\text { (multiple) }\end{array}$ & $\begin{array}{l}\text { IT specialist: Another important step is making sure that the system is performing standards. The technology } \\
\text { will need to be working. Performance and response time will need to be good. It will need to be easy to use and } \\
\text { patients will need to be satisfied with usage. In addition, if there are glitches, support will need to be available. } \\
\text { Only then will patients start using the system more, and thus their behaviours and attitudes will adjust. } \\
\text { Patient: I think that easy access to ePHR is key. Such as an online page or an application on a smart phone. It has } \\
\text { to be easy to use, easy to navigate and easy to understand. }\end{array}$
\end{tabular}

Lack of interoperability Patient: Your company is developing this way and the other company that develops a similar product for another group of physicians; there is no link between them? What is the point to have 22 systems next to each other? ${ }^{a}$

Digital divide

IT specialist: From a patient's perspective, certain patients may not have access to Internet, and they would have to rely on a caregiver to transfer their information. In a future situation where all information would be online, patients without access to Internet could be penalized by such a system.

User capacities and attitudes

Lack of health literacy

Government official: Another aspect of ePHRs would be to allow access to patient to their own health results. However, nobody knows the added value of this aspect. What is the meaning of data that is not explained? [...] Data reading could provoke anxiety or even taking the wrong decisions for a patient, which could have consequences on his health. ${ }^{a}$

Professional reluctance to use ePHRs (multiple)

Patient: Certain drawbacks have come up with this system, such as healthcare professionals not appreciating having to slow down and explain things more to patients so that they can check them off in their record book. Health professional: Not being able to fully trust the patient's objectivity is the main reason why I am not completely able to buy into the idea. This is not to say that I have $100 \%$ trust in lab exams, but I trust information that comes directly from an institution more than information handed to me by a patient.

Support of ePHRs by professionals

IT specialist: ePHR usage will grow when the health professional will decide that it will be part of their services. Thus, health professional should be for ePHRs and should be the communication channel to the patient. Without that it becomes really difficult. ${ }^{a}$

Lack of IT literacy

Patient: I am worried about people who do not have computers or who are not computer-literate, for example elderly people. Another problem could be that some practitioners may not be computer-literate either.

Patient education

Environmental factors

Government commitment to ePHRs

Patient: One element that I think could facilitate the use of ePHR is more education and awareness-building about the system. People need to know what it is and how it works, and need to be informed about the patient's and the doctor's role.

Emphasis on patients with chronic diseases

Researcher: The government should support the system financially and should also follow their own laws and ensure that patients have access to health information as stated.

IT specialist: Right now, [government agencies] are running pilot projects to understand where the greatest opportunities and they are starting to recognize that the opportunities are with those who need ePHR the most, namely the very ill, new mothers or people with chronic illnesses.

Lack of governmental policies on ePHRs

ePHR administrator: There should also be a policy in place such that patients are expected to take care of their own health and manage the system. Currently, without these two dimensions, ePHR is of limited value. [...] If we believe in patient-centred care, we should follow that through with policies and legal frameworks that support this.

Variable level of e-health implementations

Researcher: In many jurisdictions in Canada, complete health information is not even available electronically to care providers, so it is even less available to patients. Until all information is available electronically, this will be a major hurdle when it comes to putting an electronic system in place.

Legal and ethical issues

Confidentiality, privacy and security issues

Control and custody of information (barrier and facilitator)

Patient: There may be risks to confidentiality. Who manipulate ePHRs? As a patient, what I want is that my record is not lost and it is confidential, to the professionals I want. ${ }^{a}$

Government official: If the patient manages the information, there is a risk for the data since we cannot know what he will do with that information, how he will preserve it, how it will be secured, etc. [...] A political problem that I perceive is the one about ownership of medical data. The support of the record belongs to the establishment or the ministry, but the content belongs to the patient. The patient has the right at any time to his health information.

Outdated provincial legislation

IT specialist: In Ontario, there is a policy called PHIPA, which guides the responsibilities related to medical records and custody of these records. Who manages the medical record. However, it is still written in a way that it is up to the custodians when to update or release information. Of course, the patients do have full obligations to their information. But it is to the custodian to decide if the information is appropriate for the patient.

Abbreviations: ePHR, electronic personal health records; EMR, electronic medical record; IT, Information technology.

${ }^{\mathrm{a}}$ These quotes were translated from French. 